records of completion. There are a set of questions at the end of the module and the volunteers are expected to achieve a set percentage to pass the mandatory training. This evidences the learning that they have taken away on completion of the module. Those less computer literate will be offered support to complete the module within the hospice. The module is currently being piloted by a group of volunteers with the intent to roll this out to the remaining volunteers.

\section{P-34 UNLOCKING THE POTENTIAL OF VIRTUAL REALITY IN PALLIATIVE CARE}

Letizia Perna-Forrest. Royal Trinity Hospice, London UK

\subsection{6/bmjspcare-2017-hospice.61}

Background Researchers have long been interested in the physiological and psychological aspects of wellbeing. Various studies have found that Virtual Reality (VR) therapy, using computer generated environments and avatars, can have a positive effect in relieving pain in paediatric intravenous cannulation (Gold et al., 2006) and burn wound debridement (Hoffman et al., 2000), as well as in alleviating symptoms of depression (Falconer et al., 2016), anxiety (Repetto \& Riva, 2011), and Post Traumatic Stress Disorder (Rothbaum et al., 2001). To date, there is little published research about the physical and psychological impact of VR Therapy using real life environments/settings and the potential for VR to be used effectively in palliative care has not been fully investigated.

Aim Existing research on the use of still images shown to healthy subjects has concluded that personally emotive images trigger stronger positive physiological and psychological responses (Fedorovskaya et al., 2001). Our study's principal hypothesis is: 'Personalised virtual reality therapy has a positive impact on adult palliative care patients' self-reported symptoms of physical and psychological well-being and pain scores thus resulting in an increase in overall quality of life/ wellbeing.'

Methods Participants on our study will be given a VR Therapy experience lasting approximately four minutes, once a week, for four weeks. We will be obtaining quantitative data through the comparative analysis of pre- and post-session Edmonton Symptom Assessment System: Revised (ESAS-R) scores and qualitative data through set interviews that will be completed with each participant after each VR Therapy session and at the end of the project.

Results/Conclusion Positive results from this study could provide the evidence required for VR Therapy to be adopted and to be used alongside current symptom control measures provided by hospices and palliative care teams to manage symptoms at the end of life. It is our hope that this study will also give us a better understanding how VR Therapy can be used responsibly and ethically for patients across hospices and other palliative care settings.

\section{P-35 CAN VIRTUAL REALITY (VR) GUIDED MEDITATION REDUCE PAIN? A FEASIBILITY AND ACCEPTABILITY STUDY}

Sheila Popert, Harmeet Riat, Emma Hodges. St Giles Hospice, Lichfield, UK

10.1136/bmispcare-2017-hospice.62
Background Research shows that VR is effective as a tool in managing acute pain (Hoffman \& Patterson, 2000; Hoffman \& Garcia-Palacios, 2001; Hoffman \& Chambers, 2011). There is also evidence that meditation is beneficial in reducing persistent pain, that the effect is cumulative and builds overtime (Morone et al., 2008). We have developed a VR Guided Meditation app narrated by Sir David Attenborough and are planning a mixed method randomised controlled trial to examine whether an immersive meditation experience enables palliative patients to enter a meditative state more easily and achieve long term pain reduction.

Aim To test the feasibility and acceptability of using VR Guided Meditation in a hospice setting.

Specific Objectives

- Compare two types of hardware.

- Establish whether the headsets are comfortable.

- Find out if the technique has an impact on pain.

- Ascertain feedback.

Method

- Hospice patients were offered the opportunity to participate.

- The VR Gear which connects to a mobile phone was compared with an Oculus Rift which connects to a computer. Each was used for $10 \mathrm{~min}$.

- Feedback via a structured questionnaire.

Results

- Participants: six female, 12 male. Age range 33 to 84 years. Sixteen with cancer, two with neurological conditions.

- Preference for Oculus Rift was unanimous.

- All enjoyed the experience and wished to repeat it.

- All described the headsets as comfortable.

- None experienced side effects.

- All experienced a reduction in pain, ranging from $20 \%$ to complete reduction.

- Comments: 'first time in months I forgot I had pain'; ‘ could have stayed there forever'; 'wonderful'; 'so distracted I forgot my pain'; 'in another world and didn't feel a bit of pain.'

Conclusion VR Guided Meditation is effective in distracting patients from their pain and it is acceptable and feasible to use in a hospice setting. Research is needed to establish whether its use enables patients to enter a meditative state more effectively leading to longer term benefits.

\section{P-36 IMPROVING FAMILIES' EXPERIENCE THROUGH TECHNOLOGY}

Emma Aspinall. Acorns Children's Hospice

10.1136/bmjspcare-2017-hospice.63

In 2016 Acorns Hospice Trust commissioned Azeus UK, the provider of its new IT system, to produce an interactive webbased portal for use by children, young people and families. This portal is an exciting bespoke development that enhances the hospice database system. The portal reaches out to the hospice users and their families to facilitate communication and enabling them to access their own information in real time helping to ensure hospice teams are working with data that is accurate. With unique log-ins the Portal users can view their appointments, calendar and put in booking requests both 